\title{
2 Zwischenruf: „Disruption ist disruptiv“ oder das „Undenkbare denken“!
}

\author{
Heinz Lohmann
}

\section{Einführung}

Kürzlich habe ich NAO kennengelernt. Mir war der kleine Roboter von Anfang an sympathisch und ich hatte schon nach zehn Minuten ein emotionales Verhältnis zu ihm. Dabei hatte er lediglich bei einem Fachgespräch zugehört, mal einen Schritt auf den jeweils Sprechenden zugemacht, mal den Kopf zur Seite geneigt oder auch mal mit den Augen gezwinkert. Dieses kurze Erlebnis hat mir im Handumdrehen noch einmal verdeutlicht, dass Technik nicht kalt und inhuman sein muss.

Kombiniert mit intelligenter Software, kann Robotik vielmehr die immer knapper werdenden Mitarbeiter in den Gesundheitsunternehmen entlasten und zudem auch noch die Qualität verbessern. Hat bisher die individuelle Erfahrung des jeweils behandelnden Arztes die medizinische Leistung ausgemacht, wird künftig durch Einsatz digitaler Technologien das kollektive Wissen nutzbar gemacht. Big Data ist keine Science-Fiction mehr. Die lernfähige Software hat schon die ersten Erfahrungsproben bestanden. Und elektronische Auswertungen von bildgebenden Verfahren lassen menschliche Diagnostiker bereits in der Präzision der Ergebnisse in vielen Fällen hinter sich. 


\section{Digitalisierung und Wissensvermittlung}

Weit vorangeschritten ist die Digitalisierung in der Wissensvermittlung. Das gilt bei Experten und Laien gleichermaßen. Die Informationsasymmetrie der Vergangenheit hat sich schon leicht verschoben. Die Transparenz nimmt zu und damit bekommen Patienten die Chance, auch Konsumenten zu werden. Zumindest temporär können sie über die sie betreffenden Fragen ihrer Behandlung mit entscheiden. Einige von ihnen lassen sich schon heute nicht mehr alles bieten. Die bisherige Expertendominanz auf dem Gesundheitsmarkt wird langsam aber sicher aufgeweicht. Die Erfahrung aus anderen Branchen zeigt, dass auf Märkten, auf denen Konsumenten einflussreicher werden, die Produkte und Dienstleistungen mehr ins Zentrum des Interesses rücken. Dadurch wandeln sich solche Wirtschaftsbereiche von einer Institutionen- zu einer Prozessorientierung. Diese Entwicklung beginnt auf dem Gesundheitsmarkt jetzt auch Fahrt aufzunehmen. Souveräne Patienten nutzen konsequent die modernen Möglichkeiten, die die Digitalisierung ihnen bieten. Erste Anzeichen dafür lassen sich aus dem massenhaften Einsatz von Geräten zur Messung von Vitaldaten ableiten. Auch der direkte Zugriff auf Angebote der Internetmedizin verdeutlicht diesen Trend. In den kommenden Jahren wird sich die Waage noch weit mehr zugunsten der Stärkung der Position von Patienten neigen.

\section{Wandel der Gesundheitsberufe}

Kürzlich postulierte der Vorstandsvorsitzende eines norddeutschen Universitätsklinikums in einer Podiumsdiskussion apodiktisch, er halte nichts davon, wenn Computerprogramme Ärzten ihre Arbeit erklärten.

Mal abgesehen davon, dass es für manche Patienten nicht schlecht wäre, wenn die sie behandelnden Mediziner mit Hilfe intelligenter Software auf den neuesten Stand des Wissens gebracht würden, offenbart aber die zitierte Aussage ein grundlegendes Missverständnis. Wer sagt denn, dass in zehn Jahren die heute von Ärzten erledigten Arbeiten immer noch ihnen vorbehalten sein werden? Gleiches gilt für Krankenpfleger, Kaufleute, Techniker und viele andere. In der Industriellen Revolution haben elektrische Webstühle die erfahrenen Handwerker aus der Produktion fast vollständig verbannt. Viele weitere althergebrachte Berufe sind ebenfalls von der Bildfläche verschwunden oder haben sich neuen Inhalten zugewandt. Die soeben erst beginnende Digitalisierung wird unsere Gesellschaft noch viel radikaler umkrempeln und auch die Medizin „vom Kopf auf die Füße stellen“.

\section{Fazit}

Von einer disruptiven Entwicklung zu sprechen, fällt deshalb inzwischen vielen Menschen nicht mehr schwer. Die damit verbundenen Konsequenzen zu akzeptieren, aber doch. Das Undenkbare zu denken, ist eben nicht einfach. Aber es wird trotzdem eintreten und zudem enorme Chancen für die Zukunft eröffnen. Denn wenn uns der Arbeitsmarkt demnächst immer wenige Ärzte und Krankenpflegekräfte beschert, werden wir die neuen technischen Möglichkeiten dringend benötigen. Die wenigen 
2 Zwischenruf: „Disruption ist disruptiv“ oder das „Undenkbare denken“!

Fachexperten können sich dann auf das Wesentliche konzentrieren und werden nicht weiter im „Improvisationstheater" unseres tradierten Gesundheitssystems „verplempert“. Tatkräftig die Zukunft zu gestalten ist somit die Aufgabe der Führungskräfte.

Wer genug Mut und Fantasie hat, sollte sich umgehend auf den Weg machen. Die anderen werden den Webern folgen. 